\title{
Analysis of the Problem and the Treatment on Rural Revitalization in China
}

\author{
Yexia Sun* \\ Northeast Normal University \\ Changchun, Jilin Province, China
}

\begin{abstract}
The Chinese government has clearly put forward that we must prioritize the development of agriculture and rural areas. The paper firstly summarizes five parts of the rural revitalization, which are important for rural economy development of China. The Rural Revitalization Strategy covers the general requirements of thriving businesses, pleasant living environments, social etiquette and civility, effective governance, and prosperity. It is important to put in place sound systems, mechanisms, and policies for promoting integrated urban-rural development, and speed up the modernization of agriculture and rural areas. Based on the problems emerged during the implementation of the strategy, the paper then analyzes the problems and points out some solutions to improve the development of each part. At last, the paper gets a conclusion that it is the key to combine current situations in urban and rural areas and to formulate a solid and feasible program.
\end{abstract}

Keywords-rural revitalization; thriving business; social governance; ecological livability

\section{INTRODUCTION}

With the rapid development of China's economy, the GDP generated by China's agriculture has been growing steadily in the past two decades, and the per capita disposable income in rural areas has also witnessed increases year by year. However, with the speeding up of rural urbanization, in pursuit of the better living environment and better educational environment for the next generation, most young people choose to buy houses in counties or larger cities, leaving left-behind elderly and children in their rural homes. The population loss in rural areas has also led to run-down housing, making it difficult to forge ahead of infrastructure construction. Meanwhile, pollution has also become an important factor restricting rural development. Compared with cities, the lack of environmental regulation in rural areas has led to significant accumulation of industrial waste here. While rural citizens are generally not well educated, making it difficult to obtain enough attention to problems caused by pesticides, feces and agricultural wastewater. Furthermore, lacking relevant treatment facilities worsens not only the pollution level, but also farmers' living environment day by day. In addition, the decreasing social status of rural citizens also attributed to an inherent image of dirty, disorder and bad environment here. Gradually, the combination of population loss and unfavorable social understanding has marginalized here culturally. In turn, an even worse degree of the population loss was caused and a vicious circle was generated. Above up, in the implementation of the Rural Revitalization Strategy, we need to start from the five aspects, namely thriving businesses, pleasant living environments, social etiquette and civility, effective governance, and prosperity.

\section{HOW TO TAKE THRIVING BUSINESSES AS THE PRIMARY TASK}

Thriving businesses are the key point of the Rural Revitalization Strategy. With solid economic foundation, other aspects of the strategy, namely pleasant living environments, social etiquette and civility, effective governance, and prosperity can be greatly and constantly benefited and eventually realize full development of themselves. It is not only for the future of China's sustainable development, but also to meet the needs of urban and rural residents for a better life.

Ensuring the food security of our country is a precondition for thriving businesses [1]. We cannot achieve it without maintaining adequate grain production. It will serve both for the safety of the country and the fundamental role of rural areas to pay attention to agricultural production. Especially in modern times, the rapid development of cities attracts a large number of the rural laboring population, while decreased rural population brings great pressure to the development of rural areas and gets the agricultural development in difficulties. In the meantime, a large scale of cash crops is planted for the farmers' pursuit of economic benefit. When it is combined with communication barriers of agricultural production information, dull sale and shortage of agricultural products can be triggered simultaneously, causing serious loss to farmers themselves and exerting impact on China's food security to some extent.

Constructing a rural industrial system with modern agriculture as the main focus and primary, secondary and tertiary industries integrated development is the driving force of thriving businesses [2]. Simply speaking, modern agriculture is to realize regional, mechanized and scientific production and commercialized and diversified agricultural products, to analyze production capacity and market trends through data and ultimately to accomplish industrialized agriculture. Therefore, we need to cultivate new professional farmers with focus on their management ability and scientific and technological knowledge. Furthermore, the government needs to invest more and try to attract other capital investment. That is because the next step to better the backward production facilities lies on extending the agricultural industrial chain, which means to process agricultural products, expand the market and make more profit, instead of simply providing the products. In addition, each village should cultivate 
characteristic industries according to its own features, such as handicraft industry, tourism industry or rural cultural industry, so as to build a rural industrial system integrating primary, secondary and tertiary industries.

Quality and green agriculture are the requirements of thriving businesses [3]. Once the integrity system collapses, the enterprise will also go bankrupt. "Thriving" is to businesses what integrity is to enterprises. To thrive, we need to focus on the quality of products. While in recent years, the use of chemical agents such as fertilizers, pesticides and early maturity agent makes farmers afraid to eat their own produce. And thus crops are grown in different areas by farmers, some for their own food and the rest for sale. The latter, in that case, not only endangers public health, but also aggravates the crisis of trust in the whole society. Quality agriculture controls at source, by establishing the industrial system, production system and operation system of modern agriculture corresponding to high-quality development, it accelerates the construction of modern agriculture and comprehensively improves the quality and competitiveness of agriculture. While green agriculture urges to comprehensively raise the level of green development, demanding the strive to build resource-saving and environment-friendly agriculture. It encourages us to promote clean agricultural production and green development in multiple aspects and sectors, including source prevention and control, input management, production technical specifications and standardized management. For instance, farm manure and livestock manure can be used in producing to replace chemical fertilizers.

\section{COMMITTING TO PROSPERITY}

At present, China remains as the largest developing country in the world, and the trend of unbalanced development and widening development gap has not been fundamentally changed. Whether China can achieve a moderately prosperous society in an all-round way depends mainly on the poverty alleviation of poor farmers.

To achieve that, firstly, we need to put in place a basic public service system in impoverished areas, and gradually improve the rural medical and health service system in these areas. By doing so, we will ease the problems of difficult access to and high cost of medical services for the poor, actively implement the national health development strategy, advance medical care for rural residents, and improve their physical quality. In addition, poverty alleviation should be actively carried out through education, so as to provide the endogenous impetus for rural development and prevent inter-generational transmission of poverty.

Secondly, we need to promote employment services and further improve the public service system for employment in impoverished areas. We must strengthen training in employment skills, promote the employment innovation project of small loans, and develop industries tailored to local conditions by taking advantage of e-commerce and logistics platforms in combination with the Internet plus strategy. At the same time, in these areas, we need to strengthen the coverage of rural subsistence allowances and basic aged-care insurance.
Thirdly, we need to breakthrough innovation and development mechanisms and implement the strategy of targeted poverty alleviation. We will set up card for archives for impoverished villages and households throughout the country, carry out data collection and analysis on the structure, degree and causes of poverty, and establish national poverty alleviation and development information network system so as to achieve dynamic detection and management. Meanwhile, in order to facilitate the implementation and the inspection of poverty relief policy, we will innovatively establish the appraisal and restraint mechanism and formulate guidelines to improve the performance appraisal of party and government leaders in impoverished counties, so as to provide institutional guarantee for poverty alleviation.

\section{Developing Rural Civilization}

Rural civilization represents pure and clement folk customs, and the unique and natural cultural characters as well. In the cultural structure and historical process of China, rural civilization shows its unique and integrated vitality. At present, however, it has been greatly impacted by external influences, and its cultural connotation and value orientation have gradually lost the original status. The backward and broken culture has brought various negative effects on economic development and social order in rural areas. Therefore, it is of great significance for the reshaping and revitalization of rural civilization [4].

First, the leading role of socialist core values ought to be strengthened. As the core of the socialist core value system, the socialist core values represent the fundamental nature and basic characteristics of the system. They also reflect the rich connotation and practical requirements of the system, serving as its highly concise and concentrated expression. During the process of rural construction, we should actively cultivate and practice the core socialist values, guide rural culture with the mainstream socialist ideology, always ensure the correct spiritual guidance of cultural development, and clarify the future development trend of rural culture and its value orientation.

Second, we will strengthen rural education and give priority to education. To provide support to people who are returning to the countryside to start businesses and develop these areas, and to sparkle the vitality of rural development, our security systems will be improved and our supports for these systems will be intensified. The root of talents cultivation lies in education. So to promote the development of rural culture and ensure the development of people's livelihood, we will advance the construction of rural schools, raise the salaries of teachers there, guarantee our citizens' legitimate right to education, and effectively solve the problem of children in rural areas having difficulty going to school.

Third, we need to improve laws and regulations and better the legal literacy of rural citizens. We will establish detailed and concrete systems at the rural grassroots level, some village regulations and agreements, for example. In some places, contents like transforming village customs and family traditions, solidarity and mutual assistance, changing customs, respecting the old and caring for the young, understanding and abiding by laws are seriously written into village rules and 
regulations to strictly regulate the behavior of grassroots people, which is conducive to transforming local customs and civilization into people's emotional identity and behavior habits.

\section{Establishing MOdERn GOVERNANCE SYSTEM}

With a vast territory and profound culture, every place in China has its own characteristics. And adjusting measures to local conditions turns out being the most effective pattern of governance. Without such governance based on rural cultural characteristics and customs, the comprehensive revitalization of rural areas is nothing but a bubble, let alone the strategic goal of modernizing the country's governance system and capacity.

First, conduct scientific governance in light of local conditions. Due to China's vast territory, numerous ethnic groups, different cultures differences and economic gaps, relatively large differences in local customs and governance modes exist in different regions. Therefore, we should combine the local cultural background and living habits and conduct scientific governance based on the scientific theoretical system. In addition, we should take people as the center and their needs as the guidance to avoid detour, saving time and social resources.

Second, face up to the need of villager autonomy. We need to intensify the villagers' sense of autonomy and practice autonomous rural management system gradually, specific measures like establishing and perfecting the village councils, villagers council, the board of supervisors can be done. Therefore, the host role of villagers in rural governance can be highlighted and multiple subjects, which is mainly composed of villagers, can be mobilized to participate in rural autonomy. In addition, we will implement such governance methods as democratic consultation and decision-making, and make village affairs transparent, and improve villagers' enthusiasm and ability of self-governance through practice.

Third, effective governance should focus more on institutional development. Methods like New Able Villagers Governance and Good Deeds have been adopted in some places, which not only gives great credit for the villagers who have made outstanding contributions in the rural governance, but also carries forward the traditional virtues of the Chinese nation, and also guides the villagers to develop towards the good aspects such as kindness, filial piety, honesty, diligence and thrift, so as to promote the overall moral cultivation. Under the relatively loose rural corms, it is essential to increase legal publicity, popularize legal knowledge for the villagers, and enable them to learn, to understand and to abide by the law. In turn, it will be conducive to them to strengthen their awareness of rights, and to know how to maintain and exercise their rights.

\section{BUILDING UP PLEASANT RURAL LIVING ENVIRONMENTS}

As the key to the strategy, pleasant living environments is not only inspiration from the problems encountered in the development of capitalist economy, but also an urgent problem to be solved in the rural revitalization after combining with the physical truth of our country. We must uphold Xi Jinping's thought on ecological civilization as sound guidance and fundamental rules, adhere to the principle of sustainability and comprehensiveness and finally realize the modernization of agriculture and rural areas with the harmonious coexistence of man and nature [5].

Protecting the rural ecological environment is an important basis and premise of pleasant living environments. Rural areas where farmers live in compact communities and land for agricultural production are both involved in this aspect, yet unfortunately, pollution treatment in current Chinese rural areas depends basically on the natural process. What's worse, burning of straw, agricultural film residues and the excessive use of chemical fertilizers and pesticides in agricultural production have all made the ecological environment there a major obstacle to the building of a moderately prosperous society in an all-round way. Since 2005, the No. 1 central document in every year highlighted rural ecological and environmental problems. However, gaps are always there between the policies, directives and programs and their implementation. Therefore, we must strengthen the punishment intensity, clear the entity responsible and improve the supervision mechanism.

Strengthening infrastructure development in rural areas is the requirement of pleasant living environments. To achieve that, we must ensure adequate infrastructure, including educational, medical and transportation infrastructures, and public service and network facilities. The toilet revolution, which has been advocated in recent years, starts with changing the inconvenient living infrastructure in rural areas, followed by the construction of modern production infrastructure. After meeting the basic needs of life and production, spiritual and health pursuits, such as library and fitness equipment, will be further improved.

Developing rural ecological economy is the key and foundation of pleasant living environments [5]. Ecological economy, after all, is about the rational development and utilization of resources. It thus appears that an ecological economic system suitable for local conditions must be established in accordance with local resource characteristics and ecological environment advantages. In addition, we must realize the policy that lucid waters and lush mountains are invaluable assets and act on this understanding, and achieve sustainable development of the regional economy.

\section{CONCLUSION}

The implementation of the Rural Revitalization Strategy stems from the urgency of solving the issues relating to agriculture, rural areas and farmers [6]. Therefore, we must place these issues high on our agenda during the implementation, as they are the fundamental parts related to the national economy and people's livelihood under the background of major social contradictions. In fact, to implement the strategy well, we must prioritize the development of agriculture and rural areas. And building rural areas with thriving businesses, pleasant living environments, social etiquette and civility, effective governance, and prosperity is the only path to realize this strategic goal. Aiming at establishing and gradually improving the rural system and mechanism, achieving urban and rural integration, and 
ultimately accelerating the process of agricultural and rural modernization, these five aspects are mutually connected and complementary. In that case, efforts need to be done from the political, economic, cultural, ecological and other aspects of the countryside, so that the full development and the comprehensive revitalization of the rural areas can be achieved. To this end, it is the clearest and effective way for the government at all levels to implement the policy in light of local characteristics.

\section{REFERENCES}

[1] T.Wen, and Z. Yang. "The Way to Achieve Thriving Businesses in the Rural Revitalization Strategy,” Administration Reform, No. 8, 2018. (In Chinese)

[2] S. Sun. "The Key of Rural Revitalization Strategy: Giving Full Play to the Supporting Role of Industry,” Journal of Honghe University, No. 4, 2017. (In Chinese)

[3] X.h, Hu. "The Practice Path of Promoting Rural Revitalization with Thriving Businesses,” Study Monthly, 2019(2). (In Chinese)

[4] L. Chen. "Exploration of China's Characteristic Rural Revitalization Strategy in New Era," Journal of Northwest A\&F University(Social Science Edition), No. 5, 2018. (In Chinese)

[5] X. Z., Kong. "Pleasant Living Environment is the Key to Rural Revitalization,” China National Conditions and Strength, no.11, 2018. (In Chinese)

[6] J. Fan. "The Theory and Practice of Rural development Strategy," Thinking, No. 5, 2018. (In Chinese) 